\title{
A Complementary Silicon Quantum Dot-Enzyme Platform for Selective Detection of Nitroaromatics
}

\section{Compounds}

Leanne A. Milburn, Christopher Jay T. Robidillo $\dagger$, Rochelin Dalangin ††, and Jonathan G.C.

Veinot*

Department of Chemistry, University of Alberta, Edmonton, T6G 2E9, Alberta, Canada

KEYWORDS: sensor, nitroaromatics, TNT, nanocrystals, quantum dot, enzyme, explosive, nerve agent

To address the issue of poor selectivity in nanotechnology-driven, portable, nitroaromatics sensors, we have coupled a ratiometric photoluminescent sensor based on silicon quantum dots and fluorescent proteins with a colorimetric, enzyme-based sensor. Together, the sensors allow differentiation of nitroaromatic compounds - specifically, distinguishing acetylcholinergic nerve agents from the explosive compounds explored herein. The combined system can detect 2,4,6trinitrotoluene, 2,4-dinitrotoluene and 4-nitrophenol with micromolar detection limits and affords subsequent differentiation from the nitro-containing nerve agent paraoxon. This demonstrates the advantage of merging elements of materials chemistry and biochemistry to devise customized sensors which can accurately identify hazardous chemical species. 
Nitroaromatic compounds (NACs) are molecules comprised of at least one aromatic ring bearing at least one nitro $\left(-\mathrm{NO}_{2}\right)$ group. They can be categorized into several classes of hazardous materials: many NACs are high energy materials (or explosives) - examples include 2,4,6trinitrotoluene (TNT) and 2,4-dinitrotoluene (DNT) —others, like fenitrothion, parathion and paraoxon (PX) show adverse biological effects and act as nerve agents. ${ }^{1,2}$ Beyond the obvious societal threats posed by explosives and nerve agents, these and other NACs are widespread pollutants and can be environmental hazards. For example, NACs can be carcinogens, cytotoxins, endocrine disruptors and/or reproductive toxins for a variety of organisms. ${ }^{3}$ Complicating matters, NACs are generally resistant to degradation and accumulate in the environment which consequently increases the likelihood of harm to local populations. ${ }^{3}$

The potential risks noted above highlight the importance of developing cost-effective, sensitive, and selective sensors that differentiate between the different classes of NACs. While modern analytical instrumentation can identify molecular structures with precision, most are costly, lab-based systems that require extensive, specialized training to operate. ${ }^{4}$ Small, portable sensors have emerged as an important research area because they can offer rapid, inexpensive, 'in the field' NAC detection. ${ }^{5}$ Fluorescence-based, on/off sensors are attractive options that rely on energy (or electron) transfer between the active sensing media and the target analyte that results in a detectable modulation of the fluorescence intensity. ${ }^{6}$ The electron-withdrawing nature of pendant $\mathrm{NO}_{2}$ groups on aromatic structural units make NACs electron acceptors (i.e., oxidizing agents) that can predictably quench the photoemission from luminophores. Early work involving silicon nanomaterial-based photoluminescence sensors focused on porous silicon. ${ }^{7}$ Sailor et al. characterized a vapor-phase porous silicon sensor for TNT, DNT and nitrobenzene which demonstrated semi-reversible quenching. ${ }^{8}$ Subsequently, Germanenko et al. demonstrated NAC 
quenching of isolated, unfunctionalized silicon nanocrystals, or silicon quantum dots (SiQDs), prepared using laser ablation. ${ }^{9}$ Drawing on this and other foundational work involving porous silicon and SiQDs, we demonstrated that functionalized, organic-soluble SiQDs obtained from thermally-induced disproportionation of hydrogen silsesquioxane (HSQ) provided probes for detecting nitroaromatic explosives (NAEs). Next, we showed that water-soluble SiQDs prepared similarly could detect nitroaromatic nerve agents (NANAs; PX and PT). In both cases, the luminescent SiQD probes were interfaced with standard filter paper to provide a convenient strip sensor platform. ${ }^{2,10}$ In the case of the water-soluble SiQDs, we further extended our study by combining the SiQD photoluminescence response with the persistent (i.e., non-quenched) luminescence of fluorescent proteins (FPs) to afford a sensitive, ratiometric sensor. ${ }^{2}$

The obvious chemical similarities of NAEs and NANAs (Figure S1) make differentiating these hazardous chemical classes non-trivial as well as an important outstanding challenge. Typical approaches toward distinguishing these chemically similar molecules involve creating detection arrays comprised of complementary sensing species that are subsequently tested against the same analyte; this approach provides a 'fingerprint' used to identify specific analytes. ${ }^{11-13}$ Such fingerprinting systems are often complicated and require computer processing infrastructure to evaluate output data. Herein, we demonstrate a sensing platform that differentiates NAEs from NANAs using straightforward visible inspection (Scheme 1a). To achieve this important advance, we have exploited the complementary virtues of materials chemistry and biochemistry and, simultaneously, expand the scope of our water-soluble SiQD/FP sensor (see SiQD surface tailoring in Scheme 1b) by demonstrating NAE sensitivity. Subsequently, to distinguish NAEs from NANAs, we constructed a literature-inspired enzymatic acetylcholine detection assay that exploits the Trinder reaction to generate a readily detected red quinoneimine dye. ${ }^{14}$ Enzymes are highly 
specialized protein catalysts that exploit hydrogen-bonding as well as hydrophobic interactions at their active sites to engage targets with exceptionally high selectivity. In the past, enzymes have been combined and/or interfaced with nanomaterials to afford detection of microbes, ${ }^{15-17}$

(a)
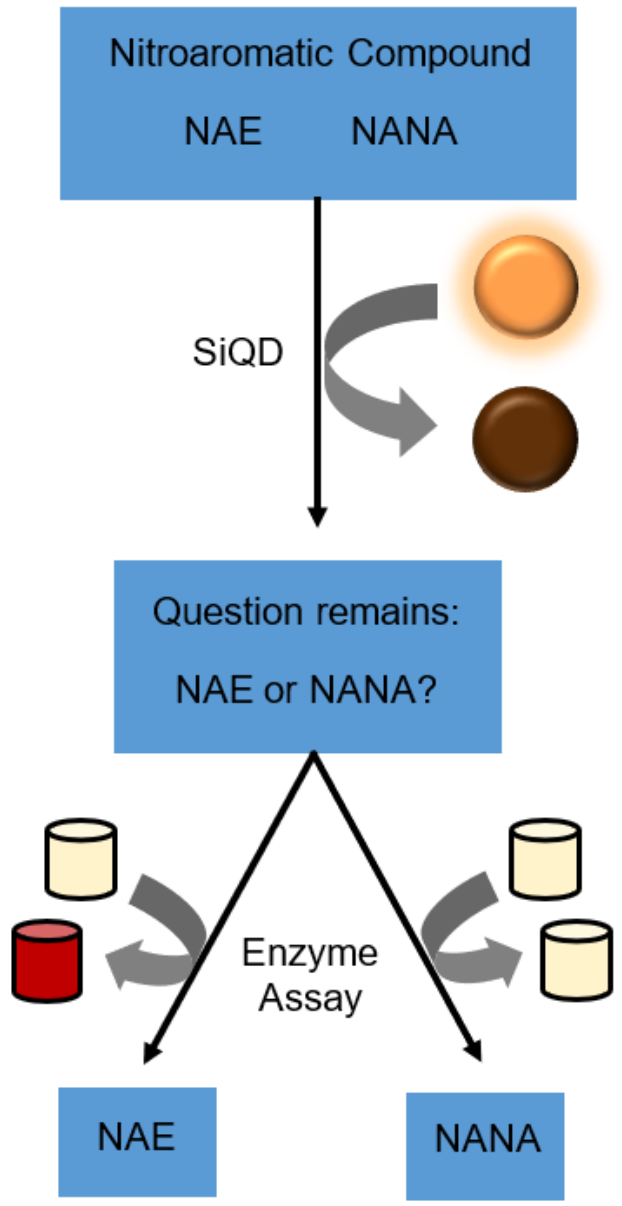

(b)

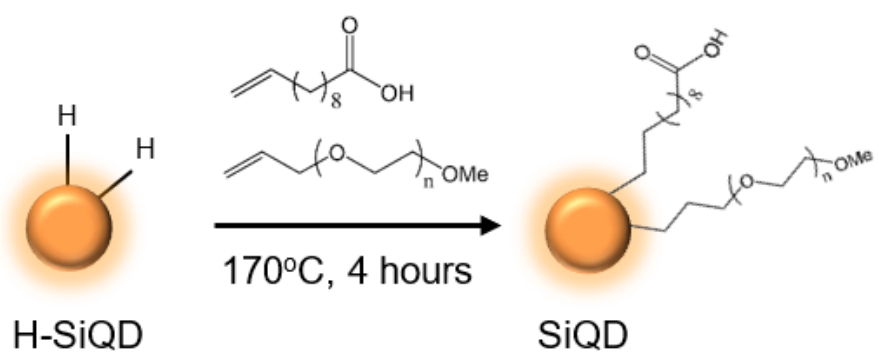

(c)

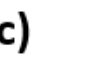

NANA

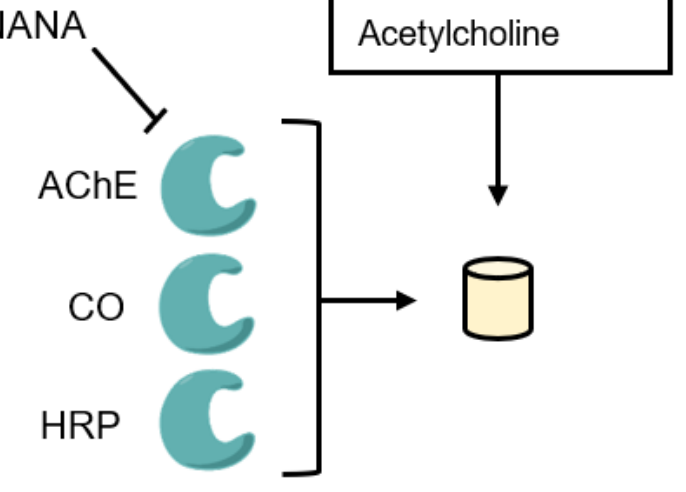

Scheme 1. a) Flow chart of the dual sensing platform presented in this work to differentiate nitroaromatic explosives (NAEs) and nerve agents (NANAs). b) Surface-functionalization of SiQDs for water-solubility. c) Assembly of the enzymatic assay involving acetylcholinesterase (AChE), choline oxidase (CO) and horseradish peroxidase (HRP). Presence of a NANA inhibits activity of $\mathrm{AChE}$, preventing dye development.

glucose, ${ }^{18,19}$ and nerve agents. ${ }^{2,20-22}$ To our knowledge, no examples have demonstrated the capacity to differentiate closely related analytes like the noted subclasses of NACs (e.g., NAEs and NANAs). For our application, acetylcholinesterase was the enzyme of interest because it is inhibited by NANAs (Scheme 1c). This allowed us to devise an enzyme-based sensor for use 
alongside our SiQD/FP sensor- the combination of these two sensing motifs provides clear differentiation between NAEs and NANAs.

The functionalized SiQDs that serve as the primary NAC detectors in the presented sensing motif were prepared using established literature procedures developed in our lab. ${ }^{23}$ A solid obtained from thermal treatment of HSQ (an amorphous silica matrix with nanocrystalline silicon inclusions) was mechanically ground and etched with alcoholic hydrofluoric acid to provide freestanding hydride-terminated SiQDs (H-SiQDs). Because detection of environmental contamination is commonly performed by evaluating water samples, and water is also a preferred solvent in public settings (e.g., airport security), we chose to employ water-soluble SiQDs in the present sensor.

To impart appropriate aqueous compatibility, H-SiQDs were functionalized with allyloxy poly(ethylene oxide) methyl ether and 10-undecenoic acid using thermally-induced hydrosilylation (Scheme 1b). ${ }^{24}$ Straightforward pairing of the functionalized SiQDs with an appropriate FP in buffered, aqueous solution provided the ratiometric sensor. The FPs of choice were determined to be mAmetrine or mCerulean3 because their photoluminescence does not overlap with that of the SiQDs (Figure S2). The interaction of the SiQDs with NAC analytes results in quenching the SiQD photoluminescence while the fluorescence of the FPs is protected by their established barrel structure and remains unaffected. ${ }^{2}$ Evaluation of the SiQD/FP combinations in 
the presence of dilute NAE solutions (i.e., TNT, DNT and 4-nitrophenol (NP)) was performed using a Molecular Devices SpectraMaxi3x photoluminescence spectrometer (Figure 1).
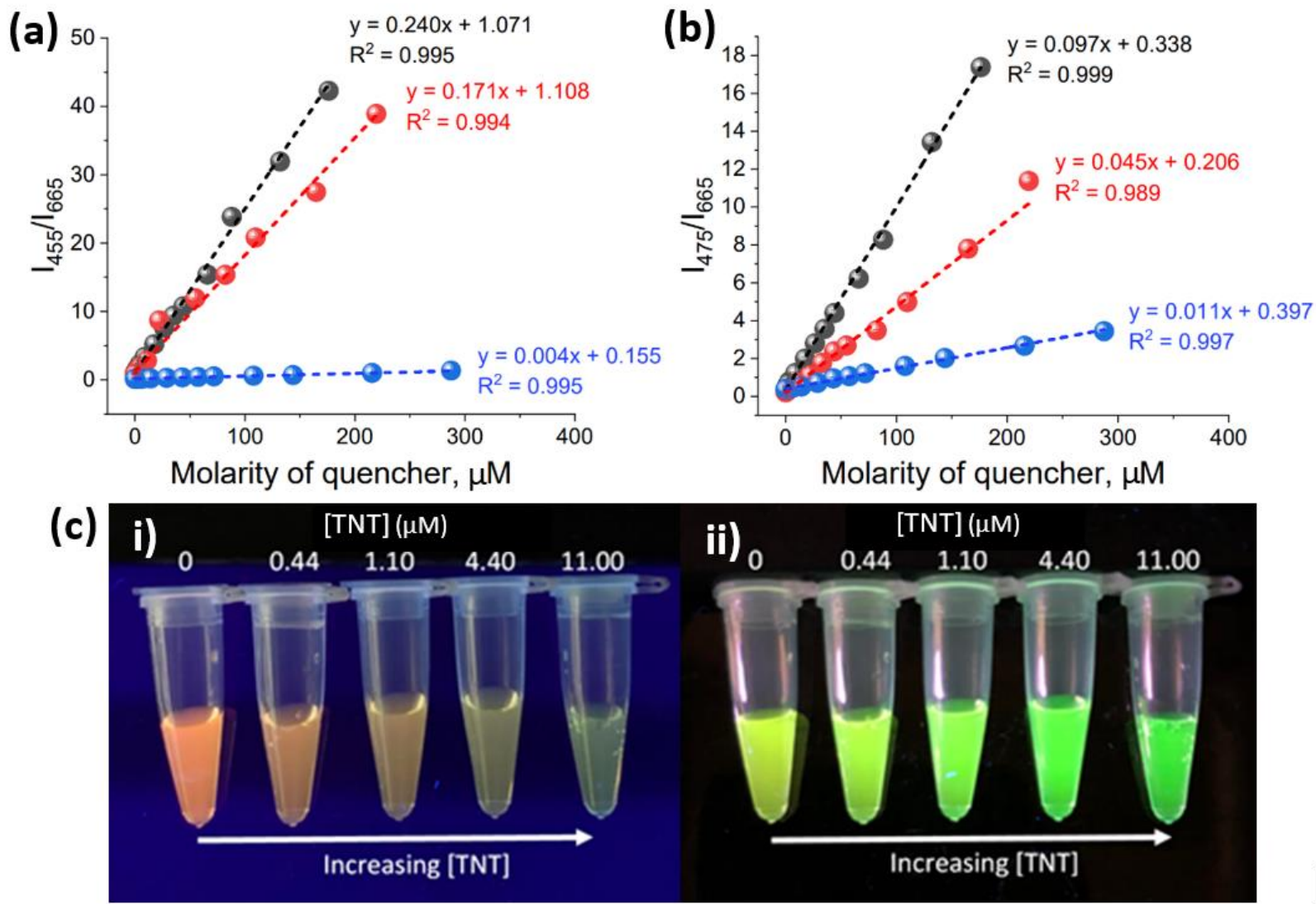

Figure 1. Representative photoluminescence (PL) quenching titration plots showing the dependence of the ratio of the photoluminescence intensities (i.e., $\mathrm{I}_{\mathrm{FP}} / \mathrm{I}_{\mathrm{SiQD}}$ ) upon excitation at $365 \mathrm{~nm}$. Analyte titrations were performed with TNT (black), DNT (red), and NP (blue) for two ratiometric sensor FP/SiQD combinations: (a) mAmetrine $\left(\lambda_{\mathrm{PL}, \max }=525 \mathrm{~nm}\right)$ and (b) mCerulean3 $\left(\lambda_{\mathrm{PL}, \max }=475 \mathrm{~nm}\right)(\mathrm{n}=4)$. (c) Photographs of the photoluminescence quenching response of the mAmetrine/SiQD sensor to TNT exposure, using (i) an excitation source that selectively excites SiQDs, and (ii) a broadband UV source that excites the mAmetrine and SiQD luminophores.

By evaluating the photoluminescence quenching of the mixtures, we determined that the SiQD photoluminescence decreases linearly with increasing TNT, DNT and NP concentrations. We also tracked quenching ratiometrically by evaluating the ratio of the SiQD and FP emissions (Figures $1 \mathrm{a}, \mathrm{b}$ ). As expected, the magnitude of the quenching response qualitatively correlates with the number of $\mathrm{NO}_{2}$ functional groups bonded to the aromatic ring of the analyte. These results 
were independent of the FP used (Figure 1a,b). Using the linear plots and a standard IUPAC equation (Equation $\mathrm{S} 2$ ), the average detection limits $\left(\mathrm{L}_{\mathrm{D}}\right)$ for the NAE were determined to be 1.9, 3.7 and $10.6 \mu \mathrm{M}$ for TNT, DNT, and NP, respectively, depending on the SiQD/FP pairing (Table S1). The present $L_{D}$ values are modest when compared with those achieved using other fluorescence quenching systems; ${ }^{25}$ this performance has previously been attributed to limited interaction between the SiQD surface and the NAE analyte and its optimization is the subject of an ongoing investigation. ${ }^{26}$

Having demonstrated that the $L_{D}$ of NAEs was consistent with that previously reported for NANAs using the same SiQD/FP system $(4.9 \mu \mathrm{M}$ for PX $),{ }^{2}$ we sought to design and incorporate the NANA-specific enzymatic assay to provide a secondary test to differentiate unknown nitroaromatics. Constructing the acetylcholine detection assay (See Supporting Information for details) in-solution verified that $\mathrm{PX}$ inhibited $\mathrm{AChE}$ activity and, thus, the formation of the red quinoneimine dye. In contrast, TNT did not influence AChE activity and the red quinoneimine dye formed. Clearly, visual detection of the appearance of the characteristic red color of the dye provides differentiation of TNT from PX and, by extension, NAEs from NANAs. To gain insight into the rapidity with which NAEs and NANA can be detected and differentiated, quinoneimine dye development was temporally tracked in the presence of PX and TNT (Figure 2). We note that the AChE population utilized in the present study can be saturated at an analyte concentration of $5 \mu \mathrm{M}$ so this concentration was used for all subsequent trials (Figure 2a). In solution phase, the development time necessary to visually distinguish $5 \mu \mathrm{M}$ PX samples from the control (PX $0 \mu \mathrm{M}$ ) is 5 minutes. For context, this response time is approximately twice as fast as most recently reported colorimetric detection systems. ${ }^{27-29}$ Of important note, and as expected, TNT did not show statistically significant inhibition (Figure 2b). 

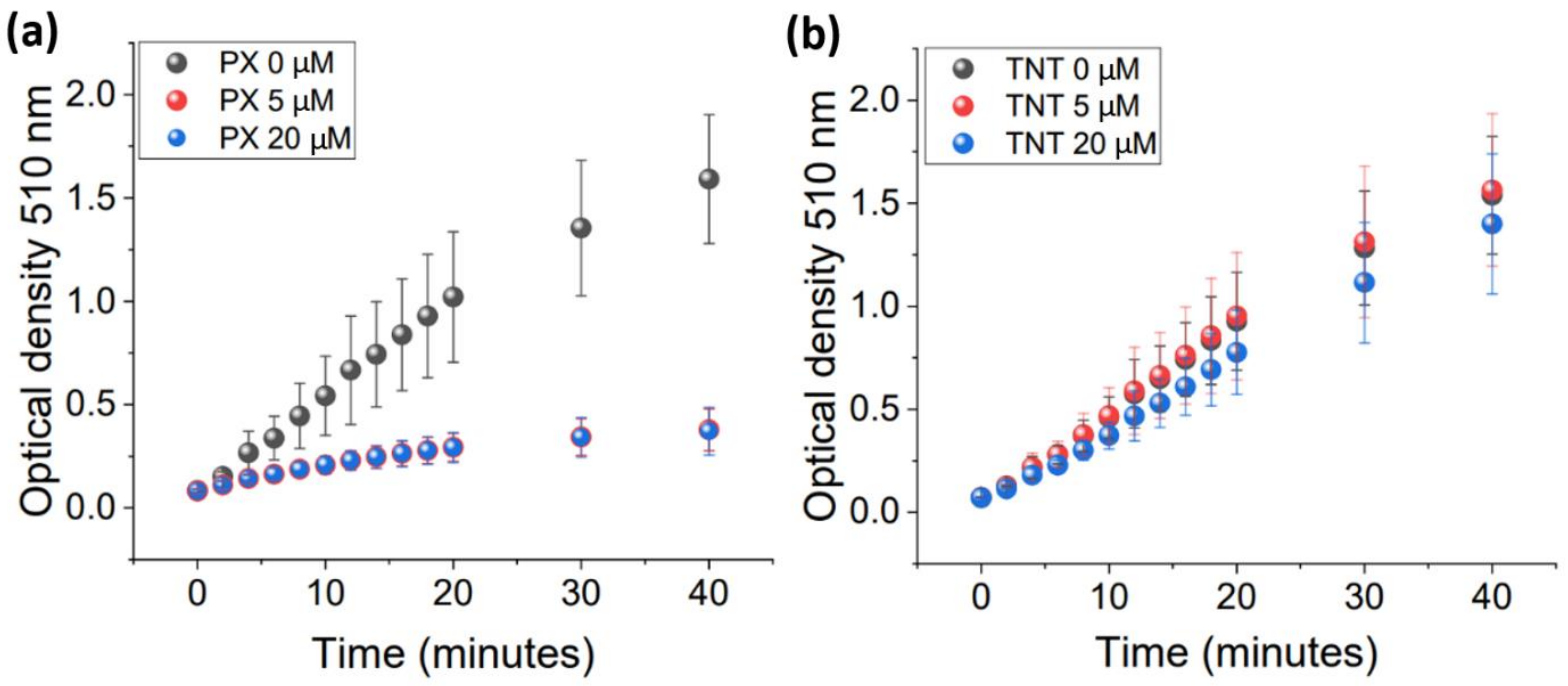

Figure 2. Solution-phase AChE inhibition trials for (a) PX and (b) TNT analytes. Inhibition is evidenced by an impairment of quinoneimine dye formation $\left(\lambda_{\mathrm{abs}, \max }=510 \mathrm{~nm}\right)$, as monitored over time. Measurements were performed in triplicate $(n=3)$, plotting the average and standard deviation.

Recognizing the well-documented instability of enzymes under ambient conditions,${ }^{30}$ we chose to address this potential limitation by immobilizing the aforementioned enzymes in an agarose gel. Our data indicate that the performance of the AChE detection assay in an agarose gel is near-identical to its performance in solution. TNT, DNT and NP behaved similarly, having no effect on the quinoneimine dye development, while PX effectively inhibited dye formation (Figure 3a,b). Importantly, the absorption spectrum after gel development highlights the selectivity of our approach and confirms that the increase in absorption at $510 \mathrm{~nm}$ can be solely attributed to the formation of the quinoneimine dye (Figure 3c). The only notable difference from the solutionbased assay, which we attribute to the increased viscosity of the gel medium, is that gelencapsulation slowed the dye development and extended the time necessary for visual differentiation of PX and TNT to approximately 10 minutes. Furthermore, the added stability 


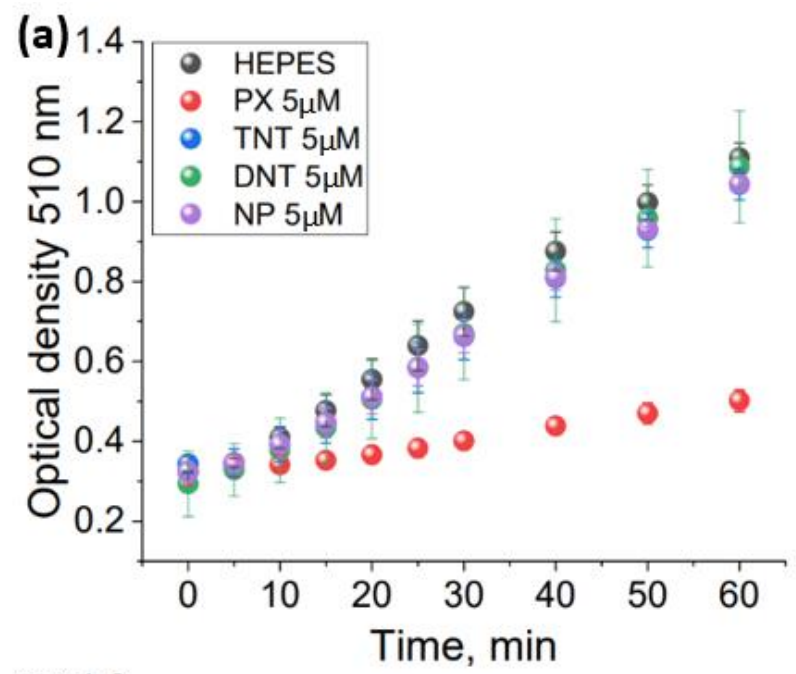

(b)
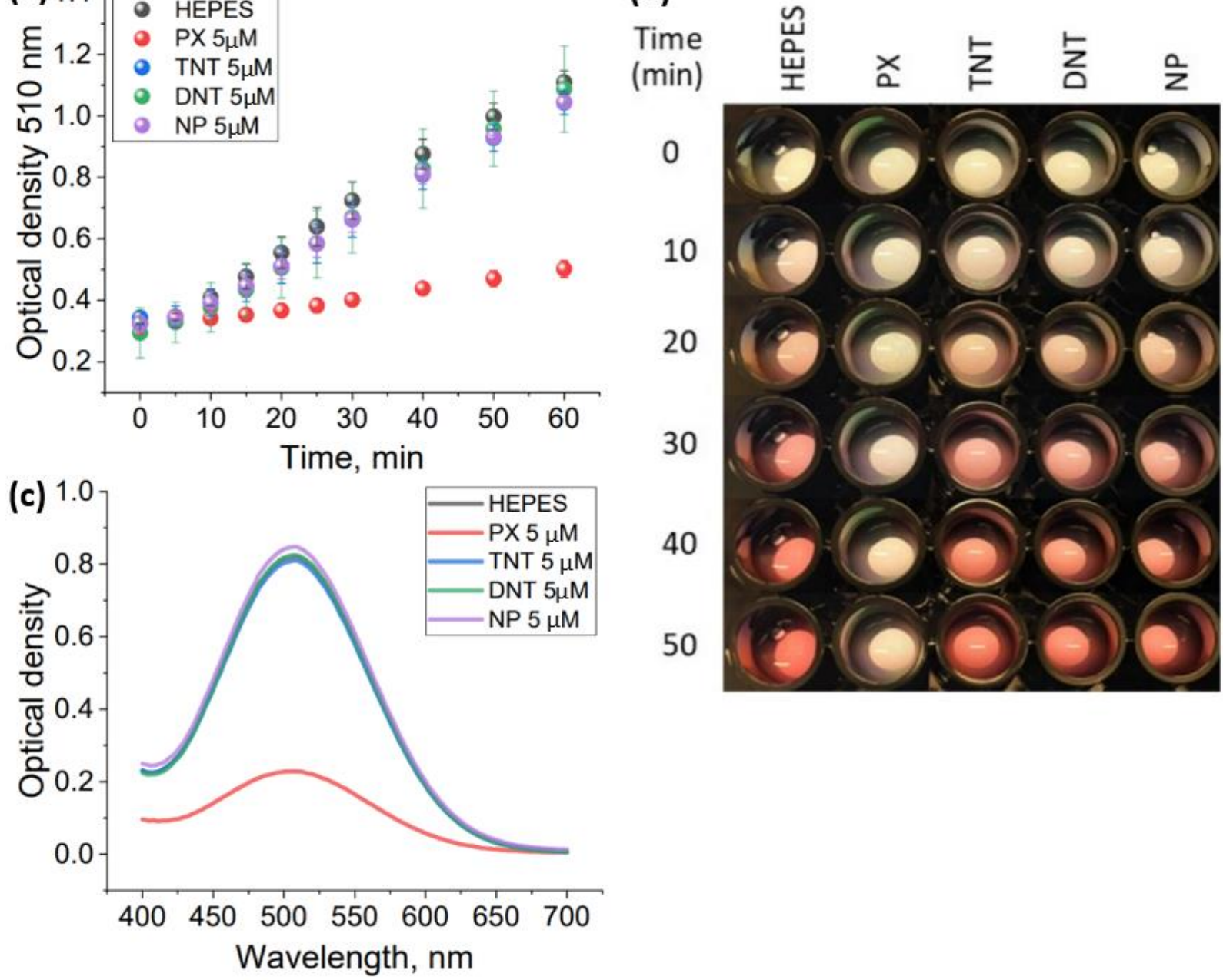

Figure 3. The gel-stabilized acetylcholine detection assay (a) expressed as the quinoneimine dye $\left(\lambda_{\mathrm{abs}, \max }=510 \mathrm{~nm}\right)$ formed over time $(\mathrm{n}=3)$, (b) a visual representation of such, and (c) the absorption spectrum after 50 minutes of development. For triplicate results, the average and standard deviation were plotted.

provided by the gel scaffold increases the longevity and portability of the assay and is expected to facilitate effective field deployment.

To summarize, we have confirmed that our aqueous $\mathrm{SiQD} / \mathrm{FP}$ photoluminescence-based sensor is sensitive to NAEs such as TNT, DNT and NP. Further, a secondary, colorimetric enzymatic assay was employed to obtain accurate and timely differentiation of hazardous nitroaromatic explosives from chemically similar nerve agents. The photoluminescent sensor 
responds immediately to indicate the presence of a nitroaromatic molecule and the colorimetric sensor provides a diagnosis of the hazard class (NAE or NANA) of the analyte within 5-10 minutes. This proof-of-concept work demonstrates the advantage introduced by combining sensing motifs from across disciplines- thus providing a portable sensing system capable of visually differentiating nitroaromatic molecules.

\section{ASSOCIATED CONTENT}

\section{Supporting Information.}

Experimental details including synthesis and functionalization of the silicon quantum dots, assembly of the acetylcholine detection assay, and titration with nitroaromatics. Calculations of averages, standard deviations, and detection limits. Chemical structures of utilized nitroaromatics. Emission spectra of silicon quantum dots and fluorescent proteins.

\section{AUTHOR INFORMATION}

Corresponding Author

*Jonathan G.C. Veinot - Department of Chemistry, University of Alberta, 11227 Saskatchewan Drive, Edmonton, Alberta, T6G 2G2, Canada; orcid.org/0000-0001-7511-510X; Email: jveinot@ualberta.ca

\section{Authors}

Leanne Milburn - Department of Chemistry, University of Alberta, 11227 Saskatchewan Drive, Edmonton, Alberta, T6G 2G2, Canada; orcid.org/0000-0001-8966-6375 
Christopher Jay T. Robidillo - Department of Chemistry, University of Alberta, 11227

Saskatchewan Drive, Edmonton, Alberta, T6G 2G2, Canada; Department of Physical Sciences and Mathematics, University of the Philippines Manila, P. Faura Street, Ermita, Manila 1000, Philippines; orcid.org/0000-0001-6492-5107

Rochelin Dalangin - Department of Chemistry, University of Alberta, 11227 Saskatchewan Drive, Edmonton, Alberta, T6G 2G2, Canada; orcid.org/0000-0002-1914-507X

Present Addresses

$\dagger$ Department of Physical Sciences and Mathematics, University of the Philippines Manila, P. Faura Street, Ermita, Manila 1000, Philippines

†† Department of Biochemistry and Molecular Medicine, University of California, Davis, Davis, CA, 95616

\section{Author Contributions}

This work was conceived of by C.J.T.R and J.G.C.V. The described synthesis, sensor assembly, and testing were performed by L.A.M. under the guidance of C.J.T.R. and J.G.C.V. Fluorescent proteins were provided by R.D. The manuscript was written by L.A.M. and J.G.C.V. All authors have reviewed and approved the final version of the manuscript.

\section{Notes}

The authors declare no competing financial interest.

\section{Acknowledgements}


The authors thank Dr. Robert E. Campbell and Gareth Lambkin for access to biological lab equipment. The Natural Science and Engineering Research Council (NSERC Discovery Grant program; RGPIN-2015-03896 and RGPIN-2020-04045), the ATUMS training program supported by NSERC CREATE (CREATE-463990-2015) as well as the University of Alberta Faculties of Science and Graduate Studies, and Alberta Innovates Strategic Projects Program, are recognized for their generous support of this project. Staff at the Analytical and Instrumentation Laboratory in the Department of Chemistry at the University of Alberta and the University of Alberta nanoFAB are thanked for their assistance with material characterization.

L.A.M. thanks NSERC for a CGS-M fellowship that has helped facilitate this project. C.J.T.R. acknowledges the University of the Philippines Manila and the University of the Philippines System Faculty, REPS, and Administrative Staff Development Program.

\section{References}

(1) Tan, C.; Nasir, M. Z. M.; Ambrosi, A.; Pumera, M. 3D Printed Electrodes for Detection of Nitroaromatic Explosives and Nerve Agents. Anal. Chem. 2017, 89 (17), 8995-9001.

(2) Robidillo, C. J. T.; Wandelt, S.; Dalangin, R.; Zhang, L.; Yu, H.; Meldrum, A.; Campbell, R. E.; Veinot, J. G. C. Ratiometric Detection of Nerve Agents by Coupling Complementary Properties of Silicon-Based Quantum Dots and Green Fluorescent Protein. ACS Appl. Mater. Interfaces 2019, 11, 33478-33488.

(3) Tiwari, J.; Tarale, P.; Sivanesan, S.; Bafana, A. Environmental persistence, hazard, and mitigation challenges of nitroaromatic compounds. Environ. Sci. Pollut. Res. 2019, 26, 2865028667.

(4) Moore, D. S. Instrumentation for trace detection of high explosives. Rev. Sci. Instrum. 2004, 75, 2499-2512. 
(5) Germain, M. E.; Knapp, M. J. Optical Explosives detection: from color changes to fluorescence turn-on. Chem. Soc. Rev. 2009, 38, 2543-2555.

(6) Gonzalez, C. M.; Veinot, J. G. C. Silicon nanocrystals for the development of sensing platforms. J. Mater. Chem. C 2016, 4, 4863.

(7) Sailor, M. J.; Wu, E. C. Photoluminescence-Based Sensing with Porous Silicon Films, Microparticles, and Nanoparticles. Adv. Funct. Mater. 2009, 19, 3195-3208.

(8) Content, S.; Trogler, W. C.; Sailor, M. J. Detection of Nitrobenzene, DNT, and TNT Vapors by Quenching of Porous Silicon Photoluminescence. Chem. - Eur. J. 2000, 6, 2205-2213.

(9) Germanenko, I. N.; Li, S.; Samy El-Shall, M. Decay Dynamics and Quenching of Photoluminescence from Silicon Nanocrystals by Aromatic Nitro Compounds. J. Phys. Chem. B 2001, 105, 59-66.

(10) Gonzalez, C. M.; Iqbal, M.; Dasog, M.; Piercey, D. G.; Lockwood, R.; Klapoetke, T. M.; Veinot, J. G. C. Detection of high-energy compounds using photoluminescent silicon nanocrystal paper based sensors. Nanoscale 2014, 6, 2608-2612.

(11) Askim, J. R.; Mahmoudi, M.; Suslick, K. S. Optical sensor arrays for chemical sensing: the optoelectronic nose. Chem. Soc. Rev. 2013, 42, 8649.

(12) Hughes, A. D.; Glenn, I. C.; Patrick, A. D.; Ellington, A.; Anslyn, E. V. A Pattern Recognition Based Fluorescence Quenching Assay for the Detection and Identification of Explosive Analytes. Chem. - Eur. J. 2008, 14, 1822-1827.

(13) Behera, P.; Mohanty, A.; De, M. Functionalized Fluorescent Nanodots for Discrimination of Nitroaromatic Compounds. ACS Appl. Nano Mater. 2020, 3, 2846-2856.

(14) Kang, H. R.; Kang, K. C.; Newby, J. G.; Lee, J. H. Fieldable flow injection analysis system with 1,1'-oxalyldiimidazole chemiluminescence detection capable of quantifying acetylcholine. Anal. Methods 2011, 3, 374-379. 
(15) Miranda, O. R.; Li, X.; Garcia-Gonzalez, L.; Zhu, Z. J.; Bunz, U. H. F.; Rotello, V. M. Colorimetric Bacteria Sensing using a Supramolecular Enzyme-Nanoparticle Biosensor. J. Am. Chem. Soc. 2011, 133 (25), 9650-9653.

(16) Chen, J.; Jiang, Z.; Ackerman, J. D.; Yazdani, M.; Hou, S.; Nugen, S. R.; Rotello, V. M. Electrochemical nanoparticle-enzyme sensors for screening bacterial contamination in drinking water. Analyst 2015, 140, 4991-4996.

(17) Duncan, B.; Le, N. D. B.; Alexander, C.; Gupta, A.; Tonga, G. Y.; Yazdani, M.; Landis, R. F.; Wang, L. S.; Yan, B.; Burmaoglu, S.; Li, X.; Rotello, V. M. Sensing by Smell: NanoparticleEnzyme Sensors for Rapid and Sensitive Detection of Bacteria with Olfactory Output. ACS Nano 2017, 11, 5339-5343.

(18) Rossi, L. M.; Quach, A. D.; Rosenzweig, Z. Glucose oxidase-magnetite nanoparticle bioconjugate for glucose sensing. Anal. Bioanal. Chem. 2004, 380, 606-613.

(19) Tian, K.; Siegel, G.; Tiwari, A. Enzymatic glucose sensor based on Au nanoparticle and plant-like ZnO film modified electrode. Mat. Sci. Eng. C 2015, 46, 548-552.

(20) Pardo-Yissar, V.; Katz, E.; Wasserman, J.; Willner, I. Acetylcholine Esterase-Labeled CdS Nanoparticles on Electrodes: Photoelectrochemical Sensing of the Enzyme Inhibitors. J. Am. Chem. Soc. 2003, 125 (3), 622-623.

(21) Simonian, A. L.; Good, T. A.; Wang, S. S.; Wild, J. R. Nanoparticle-based optical biosensors for the direct detection of organophosphate chemical warfare agents and pesticides. Anal. Chim. Acta 2005, 534 (1), 69-77.

(22) Luckham, R. E.; Brennan, J. D. Bioactive paper dipstick sensors for acetylcholinesterase inhibitors based on sol-gel/enzyme/gold nanoparticle composites. Analyst 2010, 135, 2028-2035.

(23) Clark, R. J.; Aghajamali, M.; Gonzalez, C. M.; Hadidi, L.; Islam, M. A.; Javadi, M.; Mobarok, M. H.; Purkait, T. K.; Robidillo, C. J. T.; Sinelnikov, R.; Thiessen, A. N.; Washington, J.; Yu, H.; Veinot, J. G. C. From Hydrogen Silsesquioxane to Functionalized Silicon Nanocrystals. Chem. Mater. 2017, 29, 80-89. 
(24) Robidillo, C. J. T.; Islam, M. A.; Aghajamali, M.; Faramus, A.; Sinelnikov, R.; Zhang, X.; Boekhoven, J.; Veinot, J. G. C. Functional Bioinorganic Hybrids from Enzymes and Luminescent Silicon-Based Nanoparticles. Langmuir 2018, 34, 6556-6569.

(25) Ban, R.; Zheng, F.; Zhang, J. A highly sensitive fluorescence assay for 2,4,6-trinitrotoluene using amine-capped silicon quantum dots as a probe. Anal. Methods 2015, 7, 1732-1737.

(26) Zhang, L.; Han, Y.; Zhu, J.; Zhai, Y.; Dong, S. Simple and Sensitive Fluorescent and Electrochemical Trinitrotoluene Sensors Based on Aqueous Carbon Dots. Anal. Chem. 2015, 87 (4), 2033-2036.

(27) Hu, T.; Sang, W.; Chen, K.; Gu, H.; Ni, Z.; Liu, S. Simple and sensitive colorimetric detection of a trace amount of 2,4,6-trinitrotoluene (TNT) with QD multilayer-modified microchannel assays. Mater. Chem. Front. 2019, 3, 193.

(28) Milligan, K.; Shand, N. C.; Graham, D.; Faulds, K. Detection of Multiple Nitroaromatic Explosives via Formation of a Janowsky Complex and SERS. Anal. Chem. 2020, 92, 3253-3261.

(29) Xie, X.; Xu, W.; Liu, X. Improving Colorimetric Assays through Protein Enzyme-Assisted Gold Nanoparticle Amplification. Acc. Chem. Rev. 2012, 45 (9), 1511-1520.

(30) Chapman, R.; Stenzel, M. H. All Wrapped up: Stabilization of Enzymes within Single Enzyme Nanoparticles. J. Am. Chem. Soc. 2019, 141 (7), 2754-2769. 